\title{
INTERESSI CITTADINI, FINANZE REGIE E ISTITUZIONI PARLAMENTARI NELLA SARDEGNA DEL TARDO MEDIOEVO
}

\author{
Olivetta Schena \\ Dipartimento di Storia, Beni culturali e Territorio, \\ Università di Cagliari
}

\begin{abstract}
Il saggio si propone di ricostruire, partendo dalla storiografia sul tema e attraverso l'esame degli atti dei Parlamenti del Regno di Sardegna convocati da Alfonso V nel 1421 e da Ferdinando II fra il 1481 e il 1504, il ruolo delle città all'interno dell'assemblea parlamentare, il rapporto tra le diverse realtà urbane ed il potere regio -rappresentato nell'isola dal viceré e dagli alti funzionari dell'amministrazione regia-, l'onere economico che le stesse città si assumevano con l'approvazione del donativo. La documentazione parlamentare offre uno spaccato della Sardegna reale, con i sui conflitti e con i suoi molteplici problemi, soprattutto di natura economica, ai quali danno voce le città e più in generale le componenti stamentarie che siedono in Parlamento.
\end{abstract}

Parole chiave: Regno di Sardegna, Secoli XV-XVI, Città regie, Parlamenti, Donativi.

\section{Interessos ciutadans, finances règies i institucions parlamentàries a la Sardenya de la Baixa Edat} Mitjana

Resum: Aquest assaig es proposa, a partir de la historiografia sobre el tema i a través de l'examen de les actes dels Parlaments del Regne de Sardenya convocats per Alfons V en 1421 i Ferran II entre 1481 i 1505, el paper de les ciutats al si de l'assemblea parlamentària, la relació entre les diverses realitats urbanes i el poder reial -representat a l'illa pel virrei i pels alts funcionaris de l'administració reial-, i la càrrega econòmica que les ciutats n'assumien amb l'aprovació del donatiu. La documentació parlamentària ofereix un panorama de l'autèntica Sardenya, amb els seus conflictes i els seus múltiples problemes, sobre tot de tipus econòmic, als quals donen veu les ciutats i, en general, els components estamentals del Parlament.

Paraules clau: Regne de Sardenya, Segles XV-XVI, Ciutats reials, Parlaments, Donatius.

\section{Citizen interests, royal finances and parliamentary institutions in late medieval Sardinia}

Abstract: The aim of this essay is that of retracing the role of cities within the parliamentary assembly, the relationship between the diverse urban centers and royal power -represented in the island by the viceroy and by the hierarchy of royal administration- and the economic burden, which the cities themselves used to take by approving the donative. In order to do so the essay will start from the analysis of the historiographical material on the theme and will progress through the assessment of the proceedings of the parliament of the Kingdom of Sardinia summoned by Alfonso V in 1421 and Ferdinand II between 1481 and 1504. Such parliamentary documents provide a detailed account of royal Sardinia, with its conflicts, its most diverse issues, mainly of economic nature, raised by the cities and more broadly by the representatives of the highest social classes sitting in parliament.

Key words: Kingdom of Sardinia, XVth-XVIth centuries, Royal towns, Parliament, Donations.

Data de recepció: 2 de desembre de 2014 / Data d'acceptació: 12 de febrer de 2014. 


\section{PROFILO STORIOGRAFICO}

Il ruolo delle città nella vita politica ed istituzionale del Regno di Sardegna nel Quattrocento è stato a lungo ignorato dalla storiografia. Né lo Zurita (Zurita, in Canellas López [Ed.], 1980-1990, passim) né il Fara (Fara, in Cadoni [Ed.], 1992, passim), autorevoli autori della seconda metà del Cinquecento, manifestano alcun interesse per le realtà urbane. Cagliari, Oristano, Iglesias vengono totalmente ignorate, sporadiche notizie vengono fornite su Alghero, definita opidum, ossia villa fortificata, e l'unica urbs-città degna di nota è Sassari, che -sulla scorta di quanto riportato dallo stesso Fara- instaura con la Corona un rapporto dialettico più concreto e incisivo e il cui patriziato urbano, rappresentato autorevolmente dalla nobiltà indigena di matrice comunale italiana -impersonata dai Pala, dai Gambella, dai Solinas, dai Milia e dai Marongio- manifesta direttamente a Corte, tramite suoi ambasciatori, le esigenze della città e in talune circostanze le proprie rimostranze nei confronti della politica viceregia. Ne sono un significativo esempio gli avvenimenti del 1481-1482, quando Sassari riesce a far rimuovere dall'incarico e ad allontanare dal Regno il viceré Ximén Pérez Escrivá (Fara, in Cadoni coord., 1992, 236-237).

La città di Sassari era stata beneficiata in quegli anni di numerosi privilegi, concessi da Giovanni II d'Aragona in virtù della sua generosa e decisiva partecipazione alla guerra contro Leonardo Alagòn, marchese di Oristano e visconte di Sanluri: ben 700 soldati sassaresi al comando del capitano della città, Angelo Marongio, parteciparono alla campagna militare conclusasi con la battaglia di Macomer del 1478 (Fara, in Cadoni coord., 1992, 224-225) $)^{1}$.

I Parlamenti celebrati nel Regno di Sardegna durante il regno di Ferdinando il Cattolico (1479-1516) vengono solo brevemente ricordati nell'opera del Fara, ed in essi non viene evidenziata la presenza di alcuna città regia ma ancora una volta la partecipazione prioritaria della feudalità (Fara, in Cadoni coord., 1992, 234-237, 260-261).

Il ruolo emergente ed esclusivo della classe feudale, o ceto nobiliare, che polarizza su di sé ogni interesse di studi e di ricerca, oscurando completamente la realtà urbana, è confermato fra la fine del Cinquecento e gli inizi del Seicento dalla triplice edizione, ad opera del Bellit, dell'Arquer e del Dexart dei capitoli di corte concessi allo stamento militare a conclusione dei Parlamenti svoltisi nel Regno di Sardegna nel corso dei secoli XV-XVII (Bellit, 1572; Arquer, 1591; Dexart, 1645).

Non si discosta dal Fara l'impostazione storiografica del Vico, che sembra ignorare le realtà urbane e di esse si occupa solo quando l'oligarchia cittadina si fa portavoce presso la Corte di istanze che sono certamente espressione della città in quanto tale, ma che coinvolgono soprattutto il diritto privilegiato di cui gode l'Universitas e di cui si avvantaggia il ceto nobiliare che risiede ed opera attivamente in quella città: Sassari, in particolare, è spesso protagonista di questa realtà e di queste vicende (Vico, 1639; Vico, in Manconi, 2004, passim).

Il Vico dimostra di conoscere meglio del Fara, o più semplicemente manifesta maggiore interesse per gli atti dei Parlamenti svoltisi nel Regno di Sardegna nel corso del

1 Sul diritto privilegiato di cui poté godere la città di Sassari nel corso del Quattrocento vedi Mattone, 1986a, 431-446; Schena, 1996, 205-222. 
Quattrocento, ma di essi significativamente utilizza quasi esclusivamente i capitoli di corte approvati dal sovrano su richiesta dello stamento militare: quelli stessi da lui facilmente consultabili nelle edizioni della fine del Cinquecento, curate dal Bellit e dall'Arquer. L'autore cita i ventiquattro capitoli concessi allo stamento militare nel 1511, a conclusione dell'ultimo Parlamento sardo del regno di Ferdinando II, e si sofferma ad analizzarne alcuni, a suo giudizio particolarmente significativi, ma allo stesso tempo ricorda che in quella stessa occasione il re Cattolico, preso atto della fedeltà e dei molteplici servizi resi alla Corona dalla città di Sassari, confermò ad essa gli antichi privilegi e ne accordò di nuovi (Vico, in Manconi, 2004, II, 188). Ancora una volta è Sassari che beneficia dell'attenzione dello storico, il quale sembra quasi ignorare le altre realtà urbane, che pure parteciparono attivamente ai lavori del Parlamento e ottennero l'approvazione dei capitoli sottoposti al placet del sovrano (Oliva - Schena, 1998, 164-180, 741-794), quegli stessi capitoli di corte che ai primi dell'Ottocento Giò Maria Angioy definiva significativamente "leggi fondamentali" del Regno (Mattone - Sanna, 1994, 231-308).

Un contributo decisivo allo studio delle istituzioni e del diritto vigente all'interno delle realtà urbane si ebbe alla metà dell'Ottocento con l'edizione delle fonti statutarie e di altri documenti riguardanti le città e le ville più rappresentative del Regno ad opera del Tola e del Baudi di Vesme (Tola, 1861-1868; Baudi di Vesme, 1877) cui fece seguito, fra la fine dell'Ottocento e gli inizi del Novecento, la pubblicazione sistematica degli inventari dei più importanti archivi storici cittadini: Cagliari, Iglesias, Sassari, Alghero, Oristano (Lippi, 1887 e 1902; Pinna, 1898; Era, 1927 e 1937); nonché l'edizione di alcune compilazioni manoscritte di documenti di epoca medioevale e moderna, quali: il Llibre vert di Cagliari (Di Tucci, 1925) e, in tempi a noi più vicini, il Llibre de regiment di Oristano (Ucheddu, 1998; Mele, 2007), il Libre vell, il Libre gran e il Libre de privilegis di Alghero (Manconi, 1997; Tavera - Piras, 1999; Tavera - Piras, 2007), che testimoniano inconfutabilmente la straordinaria vitalità legislativa delle realtà urbane, in quanto costituiscono il corpus normativo cittadino sedimentato nel corso dei secoli XIV-XVI e confluito nella raccolta sistematica di norme consuetudinarie, privilegi regi, prammatiche, costituzioni, capitoli di corte accolti dal sovrano nei Parlamenti e divenuti a pieno diritto leggi del Regno (Di Tucci, 1920). Queste fonti si rivelano di fondamentale importanza per la conoscenza delle istituzioni che regolavano la vita amministrativa, politica, economica e sociale delle città e delle ville sottoposte al demanio regio: dalla capitale del Regno, Cagliari, alla villa fortificata di Alghero, sottoposta definitivamente al dominio regio dal 1354 e da sempre chiave di volta per il controllo militare ed economico del Capo di Logudoro, alla meno popolosa, ma non per questo meno importante, villa di Bosa (Tasca, 1999, 2012, 2013).

\section{LE CITTÀ NEI PARLAMENTI}

L'introduzione dell'istituto parlamentare nel Regno di Sardegna rappresenta per la realtà urbana un nuovo momento di confronto con il potere regio, in quanto sin dalla prima Assemblea, convocata nel Castello di Cagliari da Pietro IV nel 1355, le città regie e le ville non infeudate venivano chiamate a partecipare ai lavori del Parlamento costituite in braccio reale (Meloni, 1993).

Già il Solmi aveva sottolineato l'interesse per la componente stamentaria del braccio reale, che rappresentava le città organizzate in municipia e le ville dipendenti direttamente 
dalla Corona, dal momento che attraverso quella componente si sarebbero potuti conoscere dati interessanti sullo sviluppo dei vari centri urbani, sul loro sistema di rappresentanza a difesa degli interessi cittadini ed oligarchici (Solmi, 1917, 363-379). Ma è il Marongiu che per la prima volta delinea compiutamente il ruolo istituzionale delle città regie in seno al Parlamento, i cui procuratori o "sindaci" confluiscono in rappresentanza nel braccio reale (Marongiu, 1979, 116-118), lo studioso tuttavia non approfondisce sufficientemente il tema delle rappresentanze cittadine: la classe di appartenenza dei portavoce delle città e delle ville convocate, il loro effettivo potere rappresentativo, se queste fossero o meno uno strumento del patriziato urbano rappresentato da nobili famiglie di origine iberica trapiantate nel Regno, come nel caso della città di Cagliari, o dalla nuova nobiltà di origine sarda, significativamente rappresentata nella città di Sassari².

Gli studi successivi, in particolare quelli condotti da Anatra e Mattone (Anatra, 1987, 164-181; Mattone, 1986b, 127-179; Mattone, 1991, 19-44), evidenziano nel panorama politico, economico ed istituzionale del Regno una forte preminenza del patriziato urbano e dell'aristocrazia terriera, entrambi di origine feudale, almeno sino alla metà del XV secolo, che costituiscono il vero ceto privilegiato, unico e diretto interlocutore della Corona nel Regno. Solo nei due secoli successivi questo "ceto privilegiato entra in crisi" e si assiste ad un suo netto ridimensionamento sia nei confronti del potere centrale sia, soprattutto, nei confronti delle forze locali, in particolare le città regie, che solo allora assumono un rapporto privilegiato con la Corona e questa "fattivamente vitalizza la presenza e il ruolo dei centri urbani di giurisdizione regia nel Regno di Sardegna” (Anatra, 1975, 35 e ss.).

Va comunque detto che per il Quattrocento manca nell'isola un potente patriziato urbano di origine mercantile, come quello barcellonese, e pertanto la "feudalità" svolse un ruolo preminente e decisivo anche nella vita parlamentare (Mattone, 1991, passim). Non a caso all'indomani della conquista del Regno le città sarde e i loro Statuti, di origine pisana o genovese, che le avevano rette nella forma perfettamente funzionale ed istituzionalizzata del comune "pazionato", vennero prontamente recepiti dalla nuova realtà politica, fatti propri dai nuovi dominatori e imbrigliati nella nuova realtà feudale (Schena, 2009, 189194). Quindi anche nel regno di Sardegna, come in altri regni dell'Italia meridionale (Napoli, Sicilia) la nobiltà cittadina rimase un élite principalmente terriera, occupata di più a difendere i propri privilegi di classe che la libera collettività cittadina (Jones, 1978, 201203). Il ruolo preminente della nobiltà feudale nel Regno di Sardegna è peraltro pienamente espresso nelle riunioni del 1447 e del 1452, che furono sessioni "parlamentari" del solo stamento militare, che si ergeva ad unico, vero garante della difesa e della sicurezza del Regno (Boscolo - Schena, 1993, 165-216; Anatra, 1975, 40-44).

Per quanto riguarda le realtà urbane, i Regni di Sardegna, Sicilia e Napoli, pur nelle significative differenze, presentano tipologie riconducibili, in linea generale, ad un quadro omogeneo. Una prima distinzione significativa, nei tre scenari individuati, va fatta tra città infeudate e città regie: molto più numerose le prime nel Mezzogiorno d'Italia, preminenti le seconde nel Regno di Sardegna. E' stato sottolineato (Galasso, 1996, 225 e ss.) come il rapporto di dipendenza dal sovrano fosse diverso per le due tipologie e come quindi l'atteggiamento regio, nei confronti delle città soggette ai feudatari e di quelle, in-

\footnotetext{
2 Anatra, 1975, p. 13, riferisce che ben quaranta titoli di nobiltà vennero conferiti a cittadini sassaresi nella prima metà del Quattrocento, e di questi almeno venticinque negli anni 1439-1444, per ragioni dettate dalla contingente esigenza di ripopolare la città e di assicurarsene la lealtà.
} 
vece, facenti parte del demanio, fosse di volta in volta, a seconda del contesto storico-politico, significativamente differente.

Il nostro interesse è volto prevalentemente alle città demaniali, la cui azione in sede parlamentare, attraverso lo stamento a loro riservato, quello reale, appare più incisiva. Molto più difficile è, invece, delineare il ruolo delle città infeudate i cui interessi venivano curati e rappresentati in Parlamento dai rispettivi feudatari. Esse, tuttavia, venivano coinvolte nelle iniziative prese in sede parlamentare per quanto atteneva, come vedremo, la raccolta delle imposte ${ }^{3}$ o per definire interventi di ristrutturazione dell'assetto urbano e del sistema difensivo, di cui poi si sarebbe fatto carico il feudatario ${ }^{4}$ o per inserire anche queste realtà nelle strategie difensive del Regno, di cui pure il Parlamento si faceva carico.

Ulteriormente articolato e diversificato era poi il comportamento regio nei confronti delle città demaniali, distinte in piccole o grandi, in relazione al peso politico che queste svolgevano in seno al Regno: per la Sardegna certamente Cagliari, in quanto capitale, ebbe maggiore rilievo, nella considerazione del sovrano, rispetto alle altre città regie, ma tale rilievo non è in alcun modo paragonabile alla "concentrazione di ruolo" ricoperto da Napoli rispetto al Regno omonimo (D’Agostino, 1979; Berengo, 1999, p. 35).

Sia Galasso, per il regno di Napoli, che Tramontana, per quanto riguarda il regno di Sicilia, richiamano l'attenzione sulla necessità di definire le città del Mezzogiorno in quanto realtà politicamente, istituzionalmente e socialmente differenti da quelle dell'Italia cetro-settentrionale (Galasso, 1996, 225-226; Tramontana, 1996, 249 e ss.). Tale esigenza si pone anche per il regno di Sardegna, che di quella realtà meridionale e catalano-aragonese faceva parte; ma per quanto sia evidente che il contesto sociale, economico e demografico delle città sarde aveva un impatto meno significativo sulla compagine del Regno di quanto non lo avessero le città meridionali, i profili giuridici ed istituzionali delle realtà urbane appaiono i medesimi.

E' opportuno sottolineare come le città del Mezzogiorno non utilizzassero per autodefinirsi nel linguaggio giuridico e usuale il termine "comune", proprio di altre realtà italiane, ma quelli di civitas o di Universitas, a seconda delle circostanze. Questi termini trovano un riscontro molto preciso nelle fonti sarde, che definiscono sempre così le realtà urbane dotate di ben precise caratteristiche.

Per quanto riguarda l'ambito sardo, le fonti utilizzano il termine Universitas in modo molto preciso e circostanziato: per esempio nei capitoli di corte votati a conclusione delle Assemblee parlamentari, che, una volta approvati dal sovrano, diventavano fonte di diritti e di doveri. Ad esempio, nelle richieste presentate da Iglesias, durante il Parlamento del 1421, la città fa una netta distinzione tra i privilegi reali concessi a la dita Universitat ed i capitoli del Breve, le franchigie, le consuetudini e gli usatici di cui godeva la dita villa. Infine nel chiedere che la città non venisse mai alienata né concessa in feudo si precisa: "vulla lo dit senyor statuir perpetualment che null temps la dita vila ne altres de son terme e de sa jurediccio no puxen esser dats ne alienats..." (Boscolo - Schena, 1993, 136-137). Riteniamo che in questa ampia giurisdizione territoriale, che comprendeva più ville, vada ricercato il significato del termine Universitas, al quale, però, non si può attribuire il con-

\footnotetext{
3 Bosa, sebbene infeudata al Villamarìn, ed Olbia, infeudata ai Maça, venivano coinvolte nelle procedure per la riscossione dell'offerta (Oliva - Schena, 1998, 190, 258).

4 In sede parlamentare, venivano definite, per Olbia, le modalità tecniche e finanziarie di intervento per ristrutturare il porto (Oliva - Schena, 1998, 126).
} 
cetto di personalità collettiva che Pietro Corrao sembra invece riconoscere alle Universitates siciliane (Corrao, 1998, 176). Il fatto che nell'ampia giurisdizione territoriale, attribuita al centro dominante, siano ricompresi anche altri centri urbani minori non ci autorizza, infatti, a riconoscere a questi ultimi una personalità giuridica preesistente, che sola consentirebbe di parlare di personalità collettiva. Il soggetto che agiva giuridicamente e che figurava negli atti era sempre e solo il centro maggiore che agiva ed operava per conto di tutti i centri a lei giurisdizionalmente soggetti (le ville di Domusnova e Villamassargia erano, ad esempio, comprese nel territorio di Villa di Chiesa, l'odierna Iglesias, e sottoposte alla sua giurisdizione).

Anche nei capitoli di corte concessi alla città di Cagliari nel Parlamento del 15041511, la supplica relativa alla riconferma di tutti i privilegis, gracies e pracmatiques, capitols viene presentata per part de la dita ciutat e universitat de Caller (Oliva - Schena, 1998, Parlamento 1504-1511, doc. 388, 748), tale specificazione deve necessariamente sottendere ad una precisa distinzione tra i due termini, che ora può sfuggire ma che doveva risultare ben chiara ai contemporanei.

Oltre ai termini di Universitas e di civitas venivano anche utilizzati quelli di villa e borgo, realtà insediative diversamente caratterizzate, sia dal punto di vista demografico che sotto il profilo istituzionale, meglio identificate e compiutamente analizzate per l'area catalana (Ferro, 1987, passim). Gli storici sardi hanno sempre ricondotto i due termini di civitas e di villa, nel primo caso, alle realtà urbane che godessero di statuti cittadini e che nello stesso tempo fossero sedi vescovili; nel secondo, a realtà che godevano solo degli statuti senza essere sede vescovile, prescindendo quindi dal fattore demografico (Boscolo, 1958, 141-147).

Attraverso l'esame dei capitoli di corte presentati dal braccio reale nei Parlamenti del Quattrocento possiamo seguire lo sviluppo delle diverse realtà da una tipologia all'altra. Nel Parlamento del 1421 erano ville, dotate quindi solo degli statuti, Alghero ed Iglesias; risultano invece città Cagliari, Sassari e Bosa. Nel Parlamento del 1481 Cagliari, Sassari e Oristano sono definite città, Alghero è ancora menzionata come villa, mentre Iglesias assume il titolo di ciutat, sebbene non ancora dotata di sede vescovile, che gli verrà attribuita solo con la riforma delle diocesi del 1503. Nelle successive riunioni parlamentari del 1497 e del 1500 Alghero viene ancora menzionata come villa, mentre nel Parlamento del 1504-1511 compare come città, avendo acquisito la sede vescovile nell'ambito della ricordata riforma delle diocesi, sancita con bolla papale da Giulio II.

Già Anatra aveva rilevato il ruolo significativo svolto, nella Sardegna del Quattrocento, dal legame tra centri urbani ed apparato ecclesiastico, sottolineando come, con il profilarsi di una oligarchia cittadina forte, si determinasse una forza centripeta che attirava a sé le strutture ecclesiastiche che nella tradizione sarda di origine giudicale erano collocate più frequentemente in sedi rurali, in sintonia con la organizzazione laica del territorio, articolata in "curatorie" (circoscrizioni amministrative in cui era suddiviso il territorio) piuttosto che nelle realtà urbane (Anatra, 1985, pp. 103-105). Agli inizi del XVI secolo, alla fine del regno di Ferdinando II, tale evoluzione poteva dirsi conclusa con l'emergere delle città sul territorio e con la definitiva collocazione delle sedi episcopali in centri urbani, muniti di mura e dotati di statuti. Tale sviluppo veniva legittimato dal progetto di Ferdinando il Cattolico di adeguamento delle circoscrizioni ecclesiastiche a quelle amministrative, magistralmente delineato da Turtas (Turtas, 1990, 717-755).

E' interessante notare come anche Galasso delinei lo stesso quadro per le città del regno di Napoli, sottolineando il ruolo significativo svolto dalla Chiesa nella definizione 
della qualità urbana. Lo studioso napoletano sottolineava come la Chiesa fosse stata fattore determinante nella definizione della identità civile e morale dei centri urbani ed in particolare di quelli che erano sedi di diocesi, attribuendo all'episcopio la valenza di segno addirittura emblematico della qualità urbana (Galasso, 1996, 231).

Il panorama sardo risulta, quindi, particolarmente complesso e variegato nell'indicazione delle diverse tipologie di insediamento, con una chiara distinzione terminologica, cui non sempre risponde un'analoga valenza giuridica. Stando agli atti parlamentari, alcuni centri minori, quali per esempio Olbia, Orosei, Longosardo, Ogliastra, Posada, Sarrabus, afferiscono allo stamento reale per quanto attiene al donativo da pagare, pur non figurando in modo attivo e propositivo durante i lavori e non presentando il loro pacchetto di richieste, poi formalizzato in capitoli di corte. Alcuni di questi centri risultano avere proprie magistrature: come Sarrabus e Ogliastra, rette da un capitano, ed Orosei, Posada ed Olbia rette da un podestà (Oliva - Schena, 1998, 191). Proprio la presenza di magistrature cittadine, unitamente alla concessione di privilegi e franchigie, avrebbe consentito, nel tempo, a queste realtà di assumere dignità urbana (Mattone, 1989, 300).

\section{CITTÀ E POTERE REGIO}

E stato da più parti rilevato (Ladero Quesada, 1996, 129-132; Galasso, 1996, 241) il ruolo assolutamente preminente e trainante che svolgeva la capitale del regno rispetto alle altre città. Tale considerazione può senza dubbio essere per certi versi estesa anche alla Sardegna dove Cagliari, capitale del Regno, svolge un ruolo particolare, sia nei confronti del potere regio che nei confronti delle altre realtà urbane 5 . Il contrasto nel Regno sardo, tra potere feudale e realtà urbane, era così forte che le città preferivano, senza esitazioni, entrare a far parte del demanio regio. In questo senso vennero presentate, nel Parlamento del 1421, richieste da parte di Iglesias e sollecitate garanzie al sovrano affinché assumesse l'impegno solenne a non infeudare la città: "que null temps la dita vila, ne altres de son terme e de sa jurediccio, no puxen esser dats ne alienats, ne en alguna manera de la sua reyal Corona separades" (Boscolo - Schena, 1993, 136-137). In altri casi, come ad esempio per Bosa, la città pagò per essere riscattata dalla soggezione feudale e tornare così in seno alla Corona: "car la dita ciutat de Bosa ab appendicis e drets de aquella no pot esser separada de la vostra reyal Corona" (Boscolo - Schena, 1993, 147; Anatra, 1987, 165; Berengo, 1999, 104-105). Nonostante ciò non si può parlare, per il regno di Sardegna, di una codificata alleanza tra potere regio e poteri cittadini, come si evince dall'esame delle richieste presentate dallo stamento reale nei Parlamenti del Quattrocento.

Nel Trecento, durante la lunga fase di conquista, protrattasi per quasi un secolo (Schena, 2012, 50-68, 521-526), i catalano-aragonesi assegnarono alle città un ruolo politico e militare di grande rilievo nell'occupazione, nella conservazione e nella difesa del Regno, attraverso un ripopolamento, a volte radicale a volte mitigato, con elementi catalani, valenzani e maiorchini. Queste nuove realtà urbane potevano contare su forti garanzie sul piano economico e commerciale e su una legislazione, di impianto catalano-aragonese,

\footnotetext{
5 Mancano per il Quattrocento studi specifici su queste tematiche che ricostruiscano, ad esempio, il rapporto tra la capitale e la massima carica dell'amministrazione regia: i viceré, che pur avendo sede a Cagliari non avevano una specifica giurisdizione sulla città (Berengo, 1999, 45-46).
} 
che ne avrebbe garantito lo status (Mattone, 1989, 301). Il ruolo preminente assegnato dai catalano-aragonesi alle città emerge anche dalla gestione, a loro favore, dell'attività agricola che venne, infatti, vincolata con franchigie e privilegi, a favore della componente cittadina che monopolizzava ogni attività commerciale all'interno delle aree urbane. Tra $\mathrm{XIV}$ e XV, infatti, la legislazione commerciale catalano-aragonese riproponeva il primato dell'economia cittadina e del mercato urbano sulle realtà agricole del Regno (Mattone, 1989, 303).

Nel Quattrocento il potere regio continuò a consolidare, tra mille difficoltà e contraddizioni, il ruolo preminente assegnato alle città nel secolo precedente. Già Martino il Giovane, nel ridisegnare il quadro giurisdizionale del Regno, favorì un equilibrio tra nuova e vecchia feudalità, tra quest'ultima e le giurisdizioni urbane, assegnando, ad esempio, un ruolo significativo a Sassari, Alghero e Bosa nello spazzare le ultime resistenze feudali nel Logudoro contro il Monteacuto, l'Anglona ed i Doria (Anatra, 1987, 160; Anatra, 1986b, 365-366).

La politica di Alfonso il Magnanimo, segnata da "l'assenteismo italiano" del sovrano quale conseguenza più immediata di un programma imperialistico, impose alla Corona significative concessioni, in funzione di un ruolo preminente della feudalità, tanto che le cariche più importanti del regno divennero appannaggio dell'alto baronaggio. Approfittando degli spazi istituzionali lasciati liberi dai Narbona, dopo la fine del regno di Arborea e l'acquisizione da parte della Corona dei relativi territori, si apriva, con il Magnanimo, una nuova fase, segnata dalla vorticosa creazione di nuovi quadri nobiliari e feudali. Significativo fu, in quegli anni, il ruolo della feudalità, sebbene le città reclamassero, in quello stesso scorcio di tempo, libertà di relazioni commerciali con i contadi, salvaguardia dall'ingerenza della burocrazia e dai vincoli feudali (Anatra, 1987, 167).

L'ascesa al trono di Ferdinando il Cattolico, nella seconda metà del Quattrocento, coincise nel regno di Sardegna con gravissime turbolenze feudali dovute all'assenteismo regio ed alle conseguenze delle concessioni feudali operate da Alfonso il Magnanimo. Superata la crisi della rivolta del marchese di Oristano e recuperata al demanio vasta parte dei suoi possedimenti, con il riequilibrio, a favore del sovrano, del rapporto tra terre infeudate e terre di realenco, Ferdinando II poté impostare il proprio progetto politico basato sul redreç, che aveva i propri capisaldi nel riassetto istituzionale e nel riordino finanziario. Tale programma, meglio precisato nelle varie istruzioni che il sovrano inviò alle massime cariche dell'amministrazione regia (Oliva - Schena, 1998, 43 ss.) ${ }^{6}$, investiva in modo significativo anche le città, nei confronti delle quali la politica fernandina fu più interventista ed attiva: uno dei punti era, infatti, la difesa delle realtà urbane dall'invadenza della feudalità non disgiunto, però, da un controllo più marcato -attraverso un riesame restrittivo di tutti i privilegi concessi dai predecessori o indebitamente ed abusivamente goduti dalle città- delle realtà urbane e della loro amministrazione finanziaria sottoposta, come quella statale, ad uno stretto controllo.

\footnotetext{
${ }^{6}$ Su questi temi vedi anche il bel saggio di Francesco Manconi (Manconi, 2001, 9-44; nuova edizione Manconi, 2012, 17-64) che utilizza, tra l'altro, una interessantissima documentazione inedita relativa al viceré Dusay.
} 


\section{IL PRELIEVO FISCALE: I DONATIVI}

La politica di redreç di Ferdinando II ebbe una notevole efficacia anche sui Parlamenti svoltisi in quel periodo, sia per quanto riguardava le procedure giuridico formali (Anatra, $1986 \mathrm{a}, 187-189)^{7}$ che per quanto atteneva all'aspetto economico e finanziario legato ai donativi ed al prelievo fiscale (Serri, 1975, 181-230; Serri, 1983, 89-129; Serri, 1986, 239-241; Stumpo, 1986, 349-351).

Con il Parlamento del viceré Ximén Pérez Escrivá, svoltosi nel 1481-85, Ferdinando II introdusse in Sardegna due novità di grande rilievo: la codificata assenza del Sovrano ai lavori parlamentari, contrariamente a quanto avveniva in precedenza, e l'introduzione di un donativo con scadenza decennale, che garantisse negli anni alla Corona un gettito fiscale certo. Anche per quanto deciso in merito all'esazione/ripartimento del donativo, il Parlamento del 1481-1485 fece testo e ad esso ci si rifece sempre, durante i Parlamenti successivi (Oliva - Schena, 1998, Parlamento 1500, doc. 8, 343-345). Allora il donativo approvato ammontava a 150.000 lire, da riscuotersi in dieci anni, mediante l'imposizione del compartimen, o taxa, un'imposizione diretta basata sulla tassazione dei fuochi: i tre stamenti concorrevano con quote annue così ripartite: 7.500 lire, pari ai tre sesti dell'intera somma, il braccio militare; 5.000 lire, pari ai due sesti, il braccio reale; 2.500 lire, pari ad un sesto quello ecclesiastico, per un totale di 15.000 lire annue. All'interno di ciascuno stamento, poi, l'imposta era basata sul computo dei fuochi, che venivano tassati in maniera differente a seconda dei diversi stamenti (Era, 1955, LXXXII, 256-257).

L'imposizione del compartiment o taxa non era, comunque, l'unico metodo per raccogliere le somme destinate al donativo. Altra procedura era, infatti, quella che imponeva dei drets: cioè l'introduzione di una o più tasse speciali, su tutte le esportazioni ed importazioni via mare. Questo criterio era stato introdotto per la prima volta in Sardegna in occasione del Parlamento presieduto da Alfonso V il Magnanimo nel 1421: in particolare allora venne prevista una imposta, su tutte le merci esportate ed importate, fatta eccezione per il frumento, l'orzo, il biscotto, il vino e la carne fresca. In quella occasione la tassa ammontava a 9 denari per libra. I bracci si impegnavano a versare alla Corona 50.000 fiorini in 5 anni, a ragione di 10.000 fiorini all'anno; un'apposita commissione veniva deputata alla raccolta del $d r e t$, i cui membri restavano in carica un solo anno, e che operava ogni anno in una diversa città del Regno: il primo e quarto anno a Cagliari, il secondo a Sassari, il terzo ad Alghero, il quinto a Bosa (Oliva, 2014, 39-42). Il dret poteva essere riscosso solo per i cinque anni previsti al pagamento del donativo offerto dagli stamenti al sovrano, assolto il quale l'imposta veniva immediatamente abolita (Boscolo - Schena, 1993, 25-27; doc. 23, 158-164).

Tra i diritti straordinari, introdotti in quegli anni, vi era anche il diritto di bolla. Si trattava di un'imposta, da riscuotersi in ben individuate città e terre regie o baronali, designate sedi di raccolta. Tale diritto veniva imposto su determinate merci, normalmente soggette a questa imposta: broccati, seta, drappi di lana, cotone, tela di fustagno ed altri tessuti

\footnotetext{
7 Durante il Parlamento dei viceré Dusay e Rebolledo (1504-1511), lo stesso Ferdinando II, interpellato sui criteri da seguire nell'esame dei greuges (Schena, 2014) e nella definizione dell'offerta (Oliva, 2014), aveva ordinato di attenersi a quanto stabilito ed attuato nel precedente Parlamento del viceré Ximén Pérez Escrivá (Era, 1955, XXVIII-XXIX, note 12-13; Oliva - Schena, 1998, 90-106).
} 
(Oliva - Schena, 1998, Parlamento 1500, doc. 1, 225-228). Lo storico del diritto Antonio Era ipotizzava l'origine ecclesiastica del diritto di bolla, dichiarata -a suo giudizio- dalla stessa denominazione e comprovata dal fatto che incorreva nella scomunica chi avesse tentato di riscuoterla oltre il termine stabilito (Era, 1954, 71); in realtà lo studio condotto da Francisco Torrella Niubó sull'imposta tessile della bolla nella Catalogna medievale (Torrella Niubó, 1954, pp. 339-358), su cui verosimilmente si modellò l'imposta riscossa nel regno di Sardegna a conclusione di alcuni Parlamenti e destinata a coprire una parte del donativo offerto alla Corona, dimostra l'infondatezza di quella ipotesi.

Negli anni a cavallo fra il XV e il XVI secolo per ben quattro volte Ferdinando II convocò in Parlamento gli stamenti del regno di Sardegna, affidandone la conduzione al viceré Giovanni Dusay e alla sua morte, avvenuta nel 1507, al Ferdinando Girón de Rebolledo.

Nel Parlamento del 1495, come apprendiamo da fonti indirette, sarebbe stata fissata la somma di 15.000 lire, da riscuotere per tre anni, per complessive 45.000 lire. Tale riscossione doveva avvenire mediante l'imposizione, con scansione triennale appunto, di due nuovi diritti: il diritto di bolla ed una speciale tassa sulle importazioni ed esportazioni (Oliva - Schena, 1998, Parlamento 1497, doc. 4, 204-209).

Il diritto di bolla, di sei denari per libra, si doveva riscuotere nelle città e terre regie, come in quelle baronali e degli heretats, oltre che nei porti e nelle marine designate sedi di raccolta. L'altro diritto imposto era una tassa sulle importazioni ed esportazioni, non dissimile da quella sperimentata nel Parlamento del 1421, che avrebbe gravato sul commercio sardo nella misura di due denari per libra. Le sedi, individuate nell'isola per la raccolta del tributo, erano dettagliatamente elencate: Cagliari, Sassari, Oristano, Sarrabus, Ogliastra, Orosei, Posada, Olbia, Longosardo, Bosa, Castelsardo ed Alghero (Oliva Schena, 1998, 111, nota 21). La destinazione da dare alla somma sarebbe stata per muraglie, armi, artiglieria e ponti; ma durante i tre anni, previsti per la distribuzione delle somme raccolte, il Sovrano poteva intervenire, per avere chiarimenti sulle iniziative prese e per predisporne altre, ritenute a suo giudizio utili per la difesa del Regno.

Tra l'impegno assunto in un primo tempo dal Parlamento generale e le reali disponibilità finanziarie della Sardegna o comunque la reale volontà contributiva degli stamenti, ci fu una notevole spaccatura. Il 30 settembre 1495, infatti, Ferdinando II commentava con il Dusay il donativo offerto dall'isola, che ammontava a sole 6.000 lire. Egli stesso rilevava, allora, che in realtà avrebbe dovuto essere maggiore ma che, tenuto conto della difficile situazione economica in cui versava la città di Cagliari e l'intero Regno, non poteva essere superiore alle 6.000 lire sarde offerte. Nonostante l'esiguità della somma, meno della metà rispetto alle 15.000 lire annue indicate nell'offerta, il Sovrano si disse soddisfatto del servey, apprezzando la diligenza dimostrata e dando quindi disposizioni affinché, al più presto, la somma stabilita venisse versata al tesoriere Gabriele Sanchez (Archivo de la Corona de Aragón di Barcellona, Cancelleria, reg. 3607, f. 203; Oliva - Schena, 1998, 112, nota 25).

La debole economia mercantile del regno di Sardegna non fu, allora, in grado di sopportare un tale peso, le conseguenze che ne derivarono furono una riduzione degli scambi commerciali, un danno economico per le dogane ed un conseguente inadempimento, verso gli impegni presi nell'offerta, per la mancata resa delle due nuove imposte. Le risoluzioni di questo breve Parlamento furono comunque oggetto di dibattito nelle Assemblee successive, nel corso delle quali i criteri di esazione del donativo vennero in tutto o in parte ripresi (Oliva - Schena, 1998, 117-127; Parlamento 1500, doc. 24, 275-277; Parlamento $1504-1511$, doc. $54,355-357)$. 
Nel 1497 il Parlamento confermò i termini dell'offerta votata nel 1495 e ricorse ancora all'esazione dei drets (diritto di bolla e tassa sulle esportazioni) per la raccolta del donativo. Su un piano generale il secondo Parlamento, riproponendo il programma contributivo approvato nel 1495, riconfermava anche la procedura basata sui drets (Oliva Schena, 1998, 113-117). Né d'altra parte vi è traccia, nelle fonti, di un dibattito tra le diverse forze convocate su questo aspetto del servizio (Oliva - Schena, 1998, Parlamento 1997, doc. 15, 217-222). L'aumento però di 7.500 lire, mediante il compartiment, secondo la usuale suddivisione: $3 / 6$ al braccio militare, $2 / 6$ per quello reale e 1/6 per l'ecclesiastico, fa ipotizzare che, verosimilmente, il dibattito, esploso poi nel terzo Parlamento (1500), dovesse già essere iniziato e che il far gravare tutto lo sforzo finanziario e contributivo sulle attività commerciali avesse già creato qualche problema (Oliva - Schena, 1998, Parlamento 1504-1511, doc. 54, 355-357).

L'ennesimo sforzo contributivo, affrontato dal regno di Sardegna con il Parlamento del 1497, che riproponeva l'offerta presentata nel 1495, articolata in 15.000 lire annue per un triennio, se per l'isola costituiva un impegno economico di non lieve entità, si rivelò, invece, ben presto insufficiente per il ruolo che la Sardegna, come del resto tutti i territori della Corona, veniva chiamata a svolgere, a causa dell'impegno politico assunto sul piano internazionale dalla Spagna (Oliva - Schena, 1998, 38-52): gli eventi militari incalzavano e si facevano pressanti anche per il gravissimo e sempre presente pericolo dei Turchi, urgeva quindi porre in stato di difesa l'isola. Già nel 1499, forse negli ultimi mesi, Ferdinando II diede nuovamente mandato al Dusay per convocare un ennesimo Parlamento, al fine di predisporre una adeguata difesa del Regno sardo nel caso che ci fosse stato, da parte dei Turchi appunto, uno sbarco o addirittura un tentativo di invasione (Era, 1954, 72; Archivo de las Cortes Españolas di Madrid, Cerdeña, Leg. 15, n. 159).

Gli stamenti in questa Assemblea fecero presente che in poco più di dieci anni i sudditi del regno di Sardegna erano stati chiamati più volte a contribuire alle spese della Corona: dal donativo versato per il Parlamento del 1485, presieduto dal viceré Ximén Pérez Escrivá, al donativo straordinario per il matrimonio di Isabella, figlia di Ferdinando II e futura regina di Portogallo (Todde, 1959-1961, 271-286), i cui versamenti cominciarono a decorrere prima ancora che si concludesse il versamento di quello ordinario del 1485; a questi andava aggiunto il donativo votato nei precedenti Parlamenti del Dusay (1495 e 1497) ed ora le richieste del Sovrano presentate in questo terzo Parlamento. Il quadro del prelievo fiscale in Sardegna già pesante era reso più grave, sottolineavano gli stamenti, dalla desolante situazione economica e sociale in cui versava l'isola, sulla quale si erano abbattute, negli ultimi tempi, annate cattive per la raccolta del frumento e gravi morie di bestiame (Oliva - Schena, 1998, Parlamento 1500, doc. 1, 225-228). Pur con queste premesse, il 17 settembre 1500 gli Stamenti si dicevano ugualmente disposti a presentare la loro offerta che riproponeva, sul piano dell'impegno economico per il Regno, lo stesso donativo del 1495, costituendo un fondo di 45.000 lire in tre anni. Tale somma sarebbe stata raccolta: metà (22.500 lire) con il metodo del compartiment tra i tre bracci, con la consueta diversificazione delle quote spettanti a ciascun braccio, con rate annuali di 7.500 lire e l'altra metà (22.500 lire) con l'imposizione del diritto di bolla, fissato a 10 denari per libra di merce, da applicarsi sui prodotti che normalmente erano soggetti a quel diritto. L'imposizione del dret e della taxa avrebbe avuto decorrenza dal $1^{\circ}$ novembre 1500 e le riscossioni del donativo sarebbero state effettuate con scadenze quadrimestrali (Oliva - Schena, 1998, doc. 8, 244-245). Totale identità vi era, invece, per quanto riguardava le 
città, ville e porti reali, baronali ed allodiali, designati sedi di raccolta dell'imposta, ma in questo terzo donativo veniva preventivamente indicato il gettito annuale previsto per le singole sedi (Oliva - Schena, 1998, 119-120; qui riproposte alle tavv. 1 e 2).

L'imposizione, esazione e distribuzione del diritto di bolla e della taxa o compartiment venivano affidate ad una commissione, analoga a quella già nominata nei precedenti Parlamenti, costituita da ventiquattro membri, come richiesto nel Parlamento del 1497, portati poi a trenta ed in seguito anche a quarantacinque (Oliva - Schena, 1998, 121, nota 54). Questi commissari, suddivisi dodici per Capo e otto per stamento, avrebbero operato con ampia autorità, potestà e giurisdizione, in accordo con il luogotenente generale, ovverosia il viceré, secondo quanto stabilito in uno specifico capitolato, articolato in cinquantaquattro punti che richiama quello del 1495; entrambi, infatti, contenevano una dettagliata normativa circa le procedure da seguire per la riscossione del diritto di bolla (Oliva - Schena, 1998, Parlamento 1500, docc. 5-13, 235-254; Oliva, 2014).

Il prelievo del donativo, basato per il 50\% sul compartiment, era il risultato di un ampio dibattito tra i parlamentari, teso a verificare i danni causati dalla sola imposizione di drets per la riscossione del donativo ed i possibili vantaggi derivanti, invece, da un servizio misto. Nell' ambito di tale dibattito tra le altre iniziative, presumibilmente prese, venne costituita una commissione ristretta composta dalla contessa di Quirra, da Pietro Maça, da Luigi di Castellvì, da Giacomo d'Aragall e da Giovanni di Santa Creu (Oliva - Schena, 1998, Parlamento 1504-1511, doc. 54, 356-357), alla quale venne assegnato l'incarico di verificare la validità economica delle procedure del 1495. Le conclusioni furono che il servey basato tutto sui drets si era dimostrato nocivo, dannoso e pericoloso: le dogane ne erano rimaste distrutte, le merci avevano disertato per tale motivo i porti sardi e gli altri diritti delle municipalità ne avevano ricevuto un gran danno. Si auspicava, quindi, l'introduzione di un servizio misto, sottolineandone i vantaggi e l'efficacia economica. Gli Stamenti avevano comunque sollevato diverse eccezioni all'introduzione, nel servizio, del compartiment, il cui precedente utilizzo risaliva al Parlamento del Pérez nel 1485.

Allo scadere del triennio di esazione del donativo del terzo Parlamento, il luogotenente generale Giovanni Dusay convocò a Cagliari, per il 7 novembre 1504, la sua quarta ed ultima Assemblea stamentaria. L'iter dei lavori fu tormentato e le richieste, circa il servizio da prestare, non furono univoche e costanti ma, nell'arco di sette anni, dal 1504 al 1511 appunto, variarono moltissimo, sia per l'entità dell'impegno finanziario richiesto sia per quanto concerne i tempi di versamento del donativo (Oliva - Schena, 1998, pp. 75$106 ; 127-143)$.

A conclusione dei lavori gli stamenti si accordarono con il sovrano per un servey di 150.000 lire articolato ancora una volta su una imposizione diretta, la taxa o compartiment dei fuochi e una indiretta, i drets, imposizione di nuovi diritti, nella proporzione di 90.000 lire e 60.000 lire, da versare in dieci anni (Oliva - Schena, 1998, doc. 345, 645648). Il servizio offerto di 150.000 lire, da pagarsi con rate annue di 15.000 , veniva così suddiviso: 139.500 lire sarebbero state destinate al Sovrano, mentre le rimanenti 10.500 lire sarebbero state trattenute, per pagare i salari a coloro che avevano prestato servizio durante i lavori parlamentari, di cui veniva riportato un dettagliato e nominativo elenco, con l'indicazione della qualifica e del rispettivo salario (Oliva - Schena, 1998, docc. 352253, 654-674).

La somma complessiva sarebbe stata così raccolta: 90.000 per imposizione diretta dei tre bracci mediante il sistema del compartiment, così ripartito: 10.000 lire a carico degli 
ecclesiastici, 48.000 a carico dei feudatari e 32.000 lire da parte delle cità. Le rimanenti 60.000 lire sarebbero state raccolte mediante l'introduzione di due imposte indirette o drets di natura straordinaria, una sulle importazioni ed esportazioni via mare, che ammontava a 3 denari per libra ed il diritto di bolla di 2 denari per libra, da applicarsi su tutti i tessuti commerciati. Per le nuove imposte introdotte, come nei precedenti Parlamenti per il diritto di bolla, venivano indicate le sedi di raccolta con il computo orientativo del gettito ricavabile: per Cagliari, Sassari e Alghero si prevedeva un introito superiore alle 1.500 lire; per Oristano e Bosa intorno alle 500 lire, mentre erano nettamente inferiori quelli previsti per Castelsardo, Olbia, Ogliastra, Orosei, Posada e Sarrabus, con solo 8 lire (Oliva - Schena, 1998, 134; qui riproposta alla tav. 3).

La documentazione relativa ai donativi votati dai Parlamenti del regno di Sardegna nella seconda metà del XV secolo, durante il regno di Ferdinando II, conferma quanto già ampiamente illustrato da Serri (Serri, 1975, 211 ss.; 219 ss.), circa il modesto contributo offerto dal Regno sardo, se rapportato alle entrate complessive della Corona. In un prospetto relativo agli ultimi ventisei anni (1485-1511), infatti, sommando le uscite di natura più varia: donativi ordinari, straordinari, spese sostenute in occasioni di gravi pestilenze o calamità naturali, la cui incidenza non è trascurabile, vediamo che le spese complessive ammontavano a sole 720.000 lire, pari a circa 257.142 ducati, per un importo annuo di circa 9.890 ducati (Oliva - Schena, 1998, 141; qui riproposta alla tav. 4) . $^{8}$

Tali somme, se raffrontate con i 75.000 scudi annui versati dalla Sicilia, i 290.000 scudi di Napoli ed i 300.000 di Milano, appaiono un contributo minimo nel contesto dell'economia della Corona di Spagna. Ma se, proiettati all'esterno, tali valori appaiono quasi irrisori, non altrettanto doveva essere nel contesto dell'economia interna del Regno.

Lo sforzo contributivo al quale in questi ventisei anni era stata sottoposta la Sardegna era enorme, rispetto alle reali possibilità dell'isola. Infatti ai dati sopra menzionati andrebbero aggiunti, contributi diversi quali imposte indirette, appalti (Serri, 1975, 208-210), oltre ad altre forme di riscossione quali decime e donativi per crociate.

Del forte prelievo fiscale, attivato durante i quattro Parlamenti dal 1495 al 1511, ben poco era destinato direttamente alla Sardegna ed all'interno di tale destinazione gli interventi erano essenzialmente di natura difensiva, con nessuna attenzione a quelli di pubblica utilità. Questo tipo di problematica non dovette essere estranea ai primi tre Parlamenti $(1495,1497,1500)$ quando l'intera cifra veniva stanziata per il Regno, ma con la limitazione che fosse destinata per opere difensive. Un tentativo, da parte degli Stamenti, di sottrarsi a tale costrittiva destinazione fu la richiesta presentata nel 1509 che 40.000 lire delle 150.000 , stanziate per il donativo, venissero utilizzate per far fronte alle diverse necessità del Regno e per coprire le spese vive del Parlamento, che ammontavano a 10.500 lire per i salari: una richiesta comune anche alle Assemblee parlamentari di altri regni iberici della Corona d'Aragona, che vivevano un momento di grave crisi economica. È il caso del regno di Valenza, che all'incirca negli stessi anni offriva al re Cattolico un donativo di 125.000 lire, da versare in cinque anni a ragione di 25.000 lire all'anno; questa somma, in realtà, non perveniva nelle mani del sovrano, in quanto lo stesso si era impegnato ad investire in censi ben 75.000 lire dell'intero donativo, mentre i restanti 10.000 annui copriva-

${ }^{8}$ Per il rapporto ducati-lire sarde si rimanda a Serri (Serri, 1975, 196-197), che indica, per il 1500, la valutazione del ducato in termini di lire sarde in circa 56 soldi. 
no le 50.000 lire destinate alla reparació de greuges e alle altre spese affrontate per il regolare svolgimento del Parlamento (Belenguer Cebrià, 1976, 191-194).

Nel regno di Sardegna, invece, il potere contrattuale dell'Assemblea veniva meno e nel corso delle ultime sedute parlamentari la parte regia riusciva ad operare una sostanziale modifica a suo favore, concedendo solo la disponibilità delle 10.500 lire per i salari e riacquisendo, invece, la piena disponibilità delle rimanenti 29.500, senza più alcun cenno ad un fondo di 40.000 lire riservato al Regno.

Nell'ultima conclusio Parlamenti infine la posizione regia fu così forte, da riuscire ad imporre agli stamenti una maggiorazione del donativo di 7.500 lire, che saliva così a 157.500 lire, oltre alla significativa ed onerosa riduzione del periodo di versamento che veniva portato da dieci a sette anni (Oliva - Schena, 1998, doc. 392, 795-805). Detratte le spese per i salari dei funzionari che avevano prestato servizio nel Parlamento (10.500 lire) e per gli ufficiali regi (7.500 lire), il Sovrano si vedeva così confermato in sette anni un donativo, al netto delle spese di gestione, di 139.500 lire. Va sottolineato che gli stamenti evidenziavano la magnanimità di questo loro atto, precisando che l'accoglimento delle due richieste era frutto de mera liberalitá y graciós servey y donatiu e che questa anomala procedura non doveva, né poteva, condizionare lo svolgimento e l'atto dell'offerta dei futuri Parlamenti: non entenem que sia hagut per acte obligatori ni exemplar per los esdevenidor.

L'esperienza del Parlamento Pérez Escrivá e, soprattutto, il travagliato svolgimento di questa Assemblea -aperta nel 1504 dal Dusay e proseguita dal 1508 dal Rebolledo-, i cui lavori si erano più volte arenati per vizio di forma e per difficoltà di natura procedurale, aveva reso più accorti gli stamenti, ormai consapevoli che le procedure di queste prime Curiae generales del regno di Sardegna -che dovevano svolgersi sul modello delle Corts catalane ed iniziavano ad assumere una propria fisionomia politica e legislativa-, avrebbero condizionato nel bene e nel male lo svolgimento delle future Assemblee.

\section{CONCLUSIONI}

Non è facile dire cosa avesse reso gli stamenti tanto disponibili e per quali motivi fosse mutato così radicalmente il loro atteggiamento nei confronti di Ferdinando II. Certamente l'operosa e retta attività degli esaminatori dei greuges (Schena, 2014, 18-24) e, soprattutto, l'accoglimento da parte del Sovrano dei capitoli di corte avevano agito positivamente su di loro, e li avevano predisposti ad accogliere le petizioni regie. Non tutte, però. La richiesta di alienazione delle "incontrade" di Quartu, Meana e Belvì, accolta dagli ecclesiastici e dai feudatari, veniva respinta dal braccio reale che si appellava direttamente al re.

E' comprensibile l'opposizione delle città regie che sui territori demaniali, a differenza di quelli propri o feudali, potevano ottenere con più facilità concessioni di pascolo, semina e approvvigionamento; mentre nei feudatari, e forse anche negli ecclesiastici, prevaleva l'interesse opposto, ed essi erano favorevoli all'alienazione di quelle "incontrade" in quanto aspiravano a nuove concessioni feudali.

In attesa delle risposte di Ferdinando II i lavori venivano rinviati al 15 novembre, ma il Parlamento poteva dirsi formalmente concluso e il Rebolledo lodava gli stamenti ed esprimeva il suo compiacimento per la fedeltà e generosità verso il sovrano, da essi concretamente dimostrata nell'atto dell'offerta (Oliva - Schena, 1998, doc. 392, 803-805). 
Lo svolgimento dei lavori dell' Assemblea, protrattisi per circa sette anni, aveva posto in evidenza la forza contrattuale della feudalità nei confronti della Corona e la sua indiscussa preminenza all'interno dello schieramento stamentario.

Un ruolo importante era stato svolto da Cagliari e da Sassari, ma i contrasti e le accese rivalità municipali fra le due principali città del Regno erano ben presto confluite nello stamento militare, determinando al suo interno una scissione fra la nobiltà del Capo di Cagliari e quella del Capo di Logudoro, poi ricompostasi in nome dei comuni interessi di ceto.

Del tutto marginale il ruolo del braccio ecclesiastico, che non perdeva occasione per denunciare la situazione di grave disagio e di profonda crisi economica in cui si dibatteva il clero sardo e ripetutamente cercava di ottenere l'esonero totale, o almeno parziale, da ogni tipo di contribuzione ordinaria e straordinaria.

L'istituzione rappresentativa sarda, che già nell'episodica riunione del 1355 e in quelle del 1421 e del 1481-1485 (ed accenni in quello solo baronale del 1452) aveva dimostrato di possedere autorità e capacità politica, durante questo lunghissimo e travagliato Parlamento aveva potuto prendere coscienza di sé e della propria forza contrattuale, acquisendo netta fisionomia e maturità giuridica (Mattone, 1986b, 133-134).

Iniziava -come ha giustamente osservato Antonio Marongiu (Marongiu, 1979, 240), che pure conosceva solo parzialmente gli atti del quarto Parlamento Dusay-Rebolledouna nuova fase, un nuovo ciclo nella vita dell'istituto parlamentare sardo.

\section{PARLAMENTO 1500}

Tavola 1. Taxa o compartiment per il braccio reale.

\begin{tabular}{|c|c|c|c|}
\hline Localita' & Fuochi & \multicolumn{2}{|c|}{ Importo (in lire e soldi) } \\
\hline Cagliari & 800 & 256 lire & \\
\hline Sassari & 2.000 & 640 lire & \\
\hline Alghero & 500 & 160 lire & \\
\hline Oristano & 400 & 128 lire & \\
\hline Iglesias & 450 & 144 lire & \\
\hline Castelsardo & 180 & 57 lire & 12 soldi \\
\hline Campidano maggiore & 515 & 164 lire & \\
\hline Campidano di Milis & 464 & 148 lire & \\
\hline Campidano di Simagis & 288 & 90 lire & \\
\hline Quarto Pirri Quartuccio & 160 & 51 lire & 4 soldi \\
\hline Barbagia di Belvi & 500 & 160 lire & \\
\hline Mandrolisai & 418 & 133 lire & 16 soldi \\
\hline Parte Ulcier & 500 & 160 lire & \\
\hline Goceano & 655 & 209 lire & 12 soldi \\
\hline Totali & 7.830 fuochi & 2.503 lire & 4 soldi \\
\hline
\end{tabular}


Tavola 2. Diritto di bolla.

\begin{tabular}{ll}
\hline Cagliari & lire 2.050 \\
Oristano & lire 650 \\
Sassari & lire 2.100 \\
Alghero & lire 1.450 \\
Castelsardo & lire 150 \\
Posada & lire 50 \\
Ogliastra & lire 200 \\
Sarrabus & lire 10 \\
Olbia-Longosardo & lire 110 \\
Orosei & lire 100 \\
Bosa & lire 650 \\
Totale & lire 7.520 \\
\hline
\end{tabular}

\section{PARLAMENTO 1504-1511}

Tavola 3. Imposizione dei due drets.

\begin{tabular}{lr}
\hline Cagliari & 1.630 lire \\
Sassari & 1.680 lire \\
Alghero & 1.160 lire \\
Oristano & 520 lire \\
Bosa & 520 lire \\
Castelsardo & 120 lire \\
Olbia & 88 lire \\
Ogliastra & 160 lire \\
Orosei & 80 lire \\
Posada & 40 lire \\
Sarrabus & 8 lire \\
& 6.006 lire \\
\hline
\end{tabular}

Tavola 4. USCITE 1485-1511 (in lire).

\begin{tabular}{llr}
\hline Donativi ordinari & $1481-1485$ & 150.000 \\
& $1495-1497$ & 45.000 \\
& 1500 & 45.000 \\
& $1504-1511$ & 150.000 \\
Donativi straordinari & 1491 & \\
& 1500 & 130.000 \\
Calamità naturali & 1504 & \\
Totale & & 200.000 \\
& & 720.000 lire \\
\hline
\end{tabular}




\section{BIBLIOGRAFIA}

ANATRA, B. (1975): "Corona e ceti privilegiati nella Sardegna spagnola", in: Anatra, B., Puddu, R., Serri, G., Problemi di storia della Sardegna spagnola, Cagliari, EDES, 35-64.

ANATRA, B. (1985): "Santa Sede e Sardegna tra Medioevo ed Età moderna", Annali della Facoltà di Magistero dell'Università di Cagliari, nuova serie, IX, 61-108.

ANATRA, B. (1986a): "Il donativo nei Parlamenti sardi", in: Istituzioni rappresentative nella Sardegna medioevale e moderna, Cagliari, CRS, 187-196 (Acta Curiarum Regni Sardiniae, 1).

ANATRA, B. (1986b): "I ceti dirigenti sassaresi nell'età aragonese e spagnola", in: Mattone, A., Tangheroni, M. (Ed.): Gli Statuti Sassaresi. Economia, Società, Istituzioni a Sassari nel Medioevo e nell'Età moderna, Sassari, EDES, 365-374.

ANATRA, B. (1987): La Sardegna dall'unificazione aragonese ai Savoia, Torino, Utet, 486 p.; estratto da Daj, J., Anatra, B., Scaraffia, L. (1984): La Sardegna medioevale e moderna (vol. X della Storia d'Italia diretta da G. Galasso), Torino, Utet.

ARQUER, J.P. (1591): Capitols de Cort del Stament militar de Sardenya, ara novament restampat, Càller, Ioan Maria Galcerino, 247 p.

BAUDI DI VESME, C. (1877): Codex Diplomaticus Ecclesiensis, Augustae Taurinorum, Regio Typographeo (Historiae Patriae Monumenta, XVII).

BELENGUER CEBRIÀ, E. (1976): València en la crisi del segle XV, Barcelona, Edicions 62 s/a., $380 \mathrm{p}$.

BELLIT, F. (1572): Capitols de Cort del Stament militar de Sardenya, Càller, Ioan Maria Galcerino, $247 \mathrm{p}$.

BERENGO, M. (1999): L'Europa delle città. Il volto della società europea tra Medioevo ed Età moderna, Torino, Einaudi, 1040 p.

BOSCOLO, A. (1958): "Il braccio reale nei Parlamenti sardi del periodo aragonese", in: Boscolo, A., Medioevo Aragonese, Padova, Cedam, 141-147.

BOSCOLO, A., SCHENA, O. (1993): I Parlamenti di Alfonso il Magnanimo (1421-1452), Cagliari, CRS, 228 p. (Acta Curiarum Regni Sardiniae, 3).

CORRAO, P. (1998): "Città ed élites urbane nella Sicilia del Tre-Quattrocento", in: Oligarquías políticas y elites económicas en las ciudades bajomedievales (siglos XIV-XVI), Revista d'Història Medieval, 9, 173-191.

D'AGOSTINO, G. (1979): La capitale ambigua. Napoli dal 1458 al 1580, Napoli, Società editrice napoletana, $306 \mathrm{p}$.

DEXART, J. (1725): Capitula sive Acta Curiarum Regni Sardiniae, 2 voll., Càlari, ex typographia nobilis Petri Borro (editio princeps: Calari 1645).

DI TUCCI, R. (1920): Istituzioni pubbliche di Sardegna nel periodo aragonese. Le fonti, Cagliari, G. Turno editore, $81 \mathrm{p}$.

DI TUCCI, R. (1925): Il libro verde della città di Cagliari, Cagliari, Società Editoriale Italiana, 492 p.

ERA, A. (1927): Le raccolte di Carte specialmente di re Aragonesi e Spagnoli (1260-1715) esistenti nell'Archivio del Comune di Alghero, Sassari, Gallizzi, 204 p.

ERA, A. (1937): Municipio di Oristano. Tre secoli di vita cittadina (1479-1720) dai documenti dell'Archivio Civico, Cagliari, Tipografia Pietro Valdès, 213 p.

ERA, A. (1954): "Storia della Sardegna durante il regno di Ferdinando II il Cattolico, I. Le vicende, II. I Parlamenti”, V Congreso de Historia de la Corona de Aragón, III: Fernando el Católico y Italia, Zaragoza, Institución "Fernando el Católico", 43-76.

ERA, A. (1955): Il Parlamento sardo del 1481-1485, Milano, Giuffrè, 279 p.

FARA, J.F. (1992): Ioannis Francisci Farae Opera, Introduzione, edizione critica, apparato e traduzione italiana a cura di Enzo Cadoni, vol. 3, De rebus Sardois, libri III-IV, Sassari, Gallizzi. 
FERRO, V. (1987): El dret públic català: les institucions a Catalunya fins al Decret de Nova Planta, Vic-Barcelona, Eumo Editorial, 606 p.

GALASSO, G. (1996): "Sovrani e città nel Mezzogiorno tardo-medioevale", in: Gensini, S. (Ed.), Principi e città alla fine del Medioevo, Pisa, Pacini, 225-247 (Centro di Studi sulla Civiltà del tardo Medioevo, 6).

JONES, Ph., (1978): "Economia e società nell'Italia medievale: la leggenda della borghesia", in: Romano, R., Vivanti, C. (Ed.), Storia d'Italia, Annali, I. Dal feudalesimo al capitalismo, Torino, Einaudi, 187-372.

LADERO QUESADA, M.A. (1996): "El ejercicio del poder real: instituciones e instrumentos de gobierno", XV Congreso de Historia de la Corona de Aragón, El poder real en la Corona de Aragón (siglos XIV-XVI), Zaragoza, Gobierno de Aragón-Departamento de Educación y Cultura, I/1, 79-140.

LIPPI, S. (1887): L'Archivio del Comune di Cagliari, Cagliari, Tipografia Pietro Valdès, 272 p.

LIPPI, S. (1902): Inventario del Regio Archivio di Stato di Cagliari e notizie delle carte conservate nei più notevoli Archivi comunali, vescovili e capitolari della Sardegna, Cagliari, Tipografia Pietro Valdès, 175 p.

MANCONI, F. (1997): Libre Vell, Cagliari, AM\&D, 239 p. (I Libri dei Privilegi della città di Alghero, 2).

MANCONI, F. (1998): "De no poderse desmembrar de la Corona de Aragón: Sardenya i Països catalans, un vincle de quatre segles", Pedralbes. Revista d'Història moderna, 18, 179-194.

MANCONI, F. (2001): "Il Regno di Sardegna da Ferdinando II a Carlo V: il lungo cammino verso la modernità", in: Sardegna e Spagna. Città e territorio tra medioevo ed età moderna, Archivio Sardo. Rivista di studi storici e sociali, nuova serie, 2, 9-44.

MANCONI, F. (Ed.), (2004): Vico, F., de, Historia general de la Isla y Reyno de Sardeña, Cagliari, CUEC, 7 voll.

MANCONI, F. (2012): "Il lungo cammino verso la modernità", in: Manconi, F., Una piccola provincia di un grande impero. La Sardegna nella Monarchia composita degli Asburgo (secoli XVXVIII), Cagliari, CUEC, 17-64.

MARONGIU, A. (1979): I Parlamenti sardi. Studio storico, istituzionale e comparativo, Milano, Giuffrè, $336 \mathrm{p}$.

MATTONE A. (1986a): "Gli Statuti sassaresi nel periodo aragonese e spagnolo", in: Mattone, A., Tangheroni, M. (Ed.), Gli Statuti Sassaresi. Economia, Società, Istituzioni a Sassari nel Medioevo e nell' Età moderna, Sassari, EDES, 431-446.

MATTONE, A. (1986b): "Centralismo monarchico e resistenze stamentarie. I Parlamenti sardi del XVI e del XVII secolo", in: Istituzioni rappresentative nella Sardegna medioevale e moderna, Cagliari, CRS, 127-179 (Acta Curiarum Regni Sardiniae, 1).

MATTONE, A. (1989): "Le città e la società urbana", in: Guidetti, M. (Ed.), Storia dei Sardi e della Sardegna, III. L'Età moderna. Dagli Aragonesi alla fine del dominio spagnolo, Milano, Jaca Book, 299-332.

MATTONE, A. (1991): “'Corts' catalane e Parlamento sardo: analogie giuridiche e dinamiche istituzionali (XIV-XVII secolo)", Rivista di Storia del Diritto Italiano, a. LXIV, 19-44.

MATTONE, A. (1996, giugno): "La storiografia giuridica dell'Ottocento e il diritto statutario della Sardegna medievale", Materiali per una storia della cultura giuridica, a. XXVI, n. 1, 67-100.

MATTONE, A., SANNA, P. (1994): "Giovanni Maria Angioy e un progetto sulla storia del 'diritto patrio' del Regno di Sardegna (1802)”, in: Studi e ricerche in onore di Girolamo Sotgiu, II, Cagliari, CUEC, 231-308.

MELE, G. (2007): Llibre de Regiment, Facsimile e traduzione; Testi di Armangué, J., D’Arienzo, L., Uccheddu, F., Oristano, INSTAR-S'Alvure, 189 p. (Facsimilia Arborensia, 1).

MELONI, G. (1993): Il Parlamento di Pietro IV d'Aragona (1355), Cagliari, CRS, 358 p. (Acta Curiarum Regni Sardiniae, 2). 
OLIVA, A.M., SCHENA, O. (1998): I Parlamenti dei viceré Giovanni Dusay e Ferdinando Girón de Rebolledo (1495, 1497, 1500, 1504-1511), CRS, Cagliari, 832 p. (Acta Curiarum Regni Sardiniae, 5).

OLIVA, A.M. (2014, dicembre): "Le commissioni per il donativo nei Parlamenti del regno di Sardegna tra tardo Medio evo ed Età moderna", RiMe, 13/2, 31-51.

PINNA, M. (1898): L'Archivio Comunale di Iglesias, Cagliari-Sassari, G. Dessì, 234 p.

SCHENA, O. (1996): "Sassari e il potere regio nei secoli XV-XVI", XV Congreso de Historia de la Corona de Aragón, El poder real en la Corona de Aragón (siglos XIV-XVI), Zaragoza, Gobierno de Aragón-Departamento de Educación y Cultura, I/3, 205-222.

SCHENA, O. (2009): "Città e tradizioni normative nella Sardegna medievale: alcune linee di ricerca”, in: Bibliografia statutaria italiana (1995-2005), Roma, Biblioteca del Senato della Repubblica, 189-211.

SCHENA, O. (2012): "The kingdom of Sardinia and Corsica", in: Gamberini, A., Lazzarini, I. (Ed.), The Italian Renaissance State, New York, Cambridge University Press, 50-68, 521-526.

SCHENA, O. (2014, dicembre): "Funzione e composizione della commissione degli 'examinadors de greuges' nei Parlamenti del regno di Sardegna (secc. XV-XVI), Prime note”, RiMe, 13/2, 9-29.

SERRI, G. (1975): "I donativi sardi nel secolo XVI", in: Anatra, B., Puddu, R., Serri, G., Problemi di storia della Sardegna spagnola, Cagliari, EDES, 181-230.

SERRI, G. (1983): "Il prelievo fiscale in una periferia povera. I donativi sardi in età spagnola", Annali della Facoltà di Magistero della Università di Cagliari, VII, 1, 89-129.

SERRI, G. (1986): "Il problema del prelievo fiscale nella Sardegna Spagnola: i donativi”, in: Istituzioni rappresentative nella Sardegna medioevale e moderna, Cagliari, CRS, 239-241 (Acta Curiarum Regni Sardiniae, 1).

SOLMI, A. (1917): Studi storici sulle istituzioni della Sardegna nel Medio Evo, Cagliari, Edizioni La Zattera, $391 \mathrm{p}$.

STUMPO, E. (1986): "Dalla finanza straordinaria alla finanza ordinaria: il problema dei donativi negli stati italiani della monarchia spagnola", in: Istituzioni rappresentative nella Sardegna medioevale e moderna, Cagliari, CRS, 349-351 (Acta Curiarum Regni Sardiniae, 1).

TASCA, C. (1999): Titoli e Privilegi dell'Antica Città di Bosa, Cagliari-Oristano, La Memoria Storica - Mythos, $398 \mathrm{p}$.

TASCA, C. (2012): Bosa città regia. Capitoli di Corte, Leggi e Regolamenti (1421-1826), Roma, Carocci, $421 \mathrm{p}$.

TASCA, C. (2013): Bosa nel tardo Medioevo. Fonti per lo studio di una città mediterranea, Cagliari, $A M \& D, 376 \mathrm{p}$.

TAVERA, B., PIRAS, G.F. (1999): Libre Gran, Cagliari, AM\&D, 557 p. (I Libri dei Privilegi della città di Alghero, 2).

TAVERA, B., PIRAS, G.F. (2007): Libre de privilegis della Città di Alghero. Libre de cerimònies della Città di Alghero, Sassari, Fondazione Banco di Sardegna, 327 p. (Raccolta di documenti editi ed inediti per la Storia della Sardegna, 9).

TODDE, G. (1959-1961): "Un donativo straordinario richiesto da Ferdinando il Cattolico", Studi Sardi, XVII, pp. 271-286.

TOLA, P. (1861-1868): Codex Diplomaticus Sardiniae, 2 tomi, Augustae Taurinorum, Regio Typographeo (Historiae Patriae Monumenta, X-XII).

TORRELLA NIUBÓ, F. (1954): "El impuesto textil de la bolla en la Cataluña medieval", Hispania, 14, 56, pp. 339-364.

TRAMONTANA, S. (1996): "Monarchia e città in Sicilia", in: Gensini, S. (Ed.), Principi e città alla fine del Medioevo, Pisa, Pacini, 249-271 (Centro di Studi sulla Civiltà del tardo Medioevo, 6).

TURTAS, R. (1990): "Erezione, traslazione e unione di diocesi in Sardegna durante il Regno di Ferdinando II d'Aragona (1479-1516)", Italia Sacra. Studi e documenti di Storia ecclesiastica, 44, 715-755. 
UCCHEDDU, F. (1998): Il "Llibre de regiment" e le pergamenne dell'Archivio Comunale di Oristano (secc. $X V$-XVII), Edizione diplomatica e note storiche, Presentazione di L. D’Arienzo, Oristano, S'Alvure, $181 \mathrm{p}$.

VICO, F. DE (1639): Historia general de la Isla y Reyno de Sardeña. Dividida en siete partes, 2 voll., Barcelona, Lorenco Déu.

ZURITA, J., Anales de la Corona de Aragón, edición preparada por A. Canellas López, voll. 5-8, Zaragoza, 1980-1990. 